\title{
The kpc-scale molecular outflows in two nearby starburst galaxies, NGC 2146 and NGC 3628
}

\author{
Tsai, An-Li* \\ Institute of Astronomy, National Central University, Taiwan \\ E-mail: altsaidastro.ncu.edu.tw
}

\section{Matsushita, Satoki}

Institute of Astronomy and Astrophysics, Academia Sinica, Taiwan

E-mail: satokidasiaa.sinica.edu.tw

\section{Kotaro, Kohno}

Institute of Astronomy, University of Tokyo, Japan

Research Center for the Early Universe, University of Tokyo, Japan

E-mail: kkohnodioa.s.u-tokyo.ac.jp

\section{Nakanishi, Kouichiro}

Department of Astronomical Science, the Graduate University for Advanced Studies, Japan E-mail: nakanisi.kenao.ac.jp

\section{Matsumoto, Hironomi}

Department of Physics, Nagoya University, Japan

E-mail: matumotoeu.phys.nagoya-u.ac.jp

Outflows and the loss of material in starburst galaxies play an important role in the understanding of starburst activity. Although ionized outflows have been observed in many starburst galaxies, molecular outflows were seldom detected in the past. Our research detected sub-kpc to kpc scale molecular outflows in two starburst galaxies, NGC 2146 and NGC 3628, which are similar to the prototype starburst galaxy M82, by using a radio interferometry telescope, Nobeyama Millimeter Array (NMA). Our deep observations show that these molecular outflows have expanding velocities of $40-200 \mathrm{~km} / \mathrm{s}$ and kinetic energies of $10^{54-55} \mathrm{erg}$. In order to see the effect of molecular outflows on starburst activities, we compare our radio data with Chandra soft X-ray data. Our results show that the mechanism to push molecular outflows is mainly contributed by the shock of the supernova explosion rather than by the thermal expansion of hot gas.

Nuclei of Seyfert galaxies and QSOs - Central engine \& conditions of star formation November 6-8, 2012

Max-Planck-Insitut für Radioastronomie (MPIfR), Bonn, Germany

\footnotetext{
* Speaker.
} 


\section{Introduction}

Molecular outflows and superbubbles, the early stage of outflows, tell us important information about starburst phenomena in galaxies. These structures are, however, observed in only a few galaxies, so that their characteristics are not well understood yet. So far, only a few molecular outflows and superbubbles in galaxies have been observed with highly accurate interferometry. One famous example is the kpc-scale molecular outflow detected from the typical starburst galaxy M82. The past deep $\mathrm{CO}(1-0)$ interferometric observations toward M82 succeeded to image the clear molecular superbubble and outflows [ [ $[$ ], and to directly measure their detailed properties, such as size, velocity, mass, etc.. However, to understand how galaxies lose molecular gas in more depth, we need larger samples. We included two more examples from nearby edge-on starburst galaxies as our case studies, NGC 2146 and NGC 3628, which have similar characteristics as M82.

NGC 2146 is a nearby $\left(17.2 \mathrm{Mpc}, 1^{\prime \prime}=80 \mathrm{pc}\right.$; [3]]), IR luminous $\left(1.2 \times 10^{11} \mathrm{~L}_{\odot}\right.$; [田]), edge-on $\left(i=63^{\circ}\right.$; [[] $)$ starburst galaxy with disturbed appearance and with $100 \mathrm{kpc}$ scale neutral hydrogen tidal tails [目]. These characteristics are very similar to those of prototypical starburst galaxy M82, which shows superbubbles and kiloparsec scale outflows. Thus it is natural to expect that NGC 2146 also have similar structures as seen in M82. There is no clear evidence of molecular bubble or outflow in NGC 2146 due to the lack of deep observation in the past.

NGC 3628 is a nearby $\left(\mathrm{D}=7.7 \mathrm{Mpc} ; 1^{\prime \prime}=37 \mathrm{pc}[\right.$ []] $)$, edge-on $\left(i=87^{\circ}\right.$ [B] $]$ ), and IR luminous $\left(\log L_{\mathrm{IR}} / \mathrm{L} \odot=10.25\right.$; [四]) galaxy. Radio observations [四] show that NGC 3628 has a circumnuclear starburst. Data from the Chandra X-ray Observatory (CXO) [圆, 团] show that NGC 3628 has an asymmetric plasma bipolar outflow with $\sim 7-10 \mathrm{kpc}$ scale in both north and south parts; optical $\mathrm{H} \alpha$ observations also show that NGC 3628 has a $\sim 10 \mathrm{kpc}$-scale warm plasma gas outflow [ $\theta$ ]. [ए0]] claimed that their $\mathrm{CO}(1-0)$ Nobeyama Millimeter Array (NMA) observations detected four expanding molecular superbubbles. However, no molecular outflow has been detected.

Our CO(1-0) NMA observation of NGC 2146 and NGC 3628 first detect sub-kpc to kpc scale molecular outflows and superbubbles. We are going to discuss their properties, their impact on NGC 3628, and their evolution by comparing with the CXO archival data.

\section{Observational and Archival Data}

\subsection{CO (1-0) Observations}

We have used the NMA, which consists of six 10-meter antennas and are located at the Nobeyama Radio Observatory (NRO), to observe the CO(1-0) emission of of NGC 2146 and NGC 3628a in the central $\sim 1^{\prime}-2^{\prime}$ region. The NGC 2146 observations were made during 2001 November to 2002 April in three configurations. The total on-source time is $\sim 44$ hours. The phase tracking center of our observation is $\alpha(\mathrm{J} 2000)=06^{\mathrm{h}} 18^{\mathrm{m}} 38^{\mathrm{s}}$ and $\delta(\mathrm{J} 2000)=78^{\circ} 21^{\prime} 24^{\prime \prime}$. The NGC 3628 observations were made during 2000 December to 2002 February in 3 different configurations. The total on-source time is $\sim 40$ hours. The phase tracking center is $\alpha(\mathrm{J} 2000)=$ $11^{\mathrm{h}} 20^{\mathrm{m}} 17^{\mathrm{s}}$ and $\delta(\mathrm{J} 2000)=13^{\circ} 35^{\prime} 20^{\prime \prime}$.

The data were calibrated using the NRO software package "UVPROC II" and were CLEANed using standard procedures implemented in the NRAO software AIPS. The maps were made with natural weighting with a final synthesized beam size of $\sim 3^{\prime \prime}$. 


\subsection{Soft X-ray Archival Data}

The soft X-ray data of NGC 2146 is obtained from our collaborator, Inui, who observed NGC 2146 during 2002 August to December [四]. The target was observed six times, through 2002 August to December, with the Advanced CCD Imaging Spectrometer (ACIS) on board the CXO. The total exposure time is $60 \mathrm{ks}$ in six observations. The soft X-ray data of NGC 3628 is obtained from the CXO archive, based on the original observation by Strickland et al. in 2000 December 2 with $60 \mathrm{ks}$ total exposure time. The diffuse gas images of NGC 3628 have been published in [9], but the detail properties have not yet been discussed. In order to understand the properties of the diffuse gas, such as density, mass, energy, and pressure, we reprocessed the data, extracted the spectra, and performed model fittings.

We reprocessed the data in order to obtain the plasma properties making use of the same dataanalysis process for both galaxies. The reprocessing was done with the Chandra Interactive Analysis of Observations software package (CIAO) version 4.2, and the Chandra Calibration Database (CALDB) version 4.2.2, released on 19 April 2010. We used the High Energy Analysis software (HEAsoft) version 6.5, and the X-Ray Spectral Fitting Package (XSPEC) version 12.4 for further analysis.

\section{Results and Discussion}

\subsection{The Properties and Kinematics of Molecular Outflows}

The $\mathrm{CO}(1-0)$ data traces the molecular gas. Figure $\square$ and Figure $\square$ show the integrated intensity map and the velocity map of two starburst galaxies, NGC 2146 and NGC 3628, respectively. The

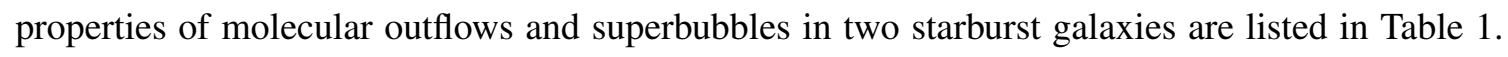
In both galaxies, we first detected sub-kpc to kpc scale molecular superbubbles or outflows with expanding velocity $40-200 \mathrm{~km} \mathrm{~s}^{-1}$. The kinetic energy of molecular superbubbles or outflows is $\sim 10^{54-55} \mathrm{erg}$, which corresponds to tens of thousands supernova explosions. The mass of molecular superbubbles or outflows is $\sim 10^{7-8} \mathrm{M}_{\odot}$, which corresponds to $1-10 \%$ of the total molecular gas in each galaxy. The expanding timescale of $\sim 10^{6-7} \mathrm{yr}$. Therefore, the mass loss rate through molecular superbubbles or outflows is $4-34 \mathrm{M}_{\odot} \mathrm{yr}^{-1}$.

Table 1: The properties of the molecular outflows and superbubbles

\begin{tabular}{lcccccccc}
\hline Galaxy & & $\begin{array}{c}\text { Size } \\
{[\mathrm{pc}]}\end{array}$ & $\begin{array}{c}v_{\text {expanding }} \\
{\left[\mathrm{km} \mathrm{s}^{-1}\right]}\end{array}$ & $\begin{array}{c}t_{\text {expanding }} \\
{[\mathrm{Myr}]}\end{array}$ & $\begin{array}{c}\text { Mass } \\
{\left[10^{8} \mathrm{M}_{\odot}\right]}\end{array}$ & $\begin{array}{c}E_{\text {kinetic }} \\
{\left[10^{54} \mathrm{erg}\right]}\end{array}$ & $\begin{array}{c}\text { Mass Loss Rate } \\
{\left[\mathrm{M}_{\odot} \mathrm{yr}^{-1}\right]}\end{array}$ & Reference \\
\hline NGC 2146 & OF1 & 2000 & $0-200$ & $10-20$ & 3.4 & 30 & $17-34$ & [ए2] \\
& SB1 & $800-1200$ & $40-60$ & $13-29$ & 2.6 & $4-9$ & $9-20$ & [ए2] \\
NGC 3628 & OF & $350-500$ & $80-100$ & $3-7$ & 0.3 & $2-3$ & $4-10$ & [ए3] \\
\hline
\end{tabular}

\subsection{The Mechanism to Push Molecular Outflows Outward}

The soft X-ray data traces the hot ionized gas. Figure B shows the comparison between molecular outflows and hot ionized gas. In NGC $2146 \mathrm{OF}$, the ionized gas is embedded inside of the 

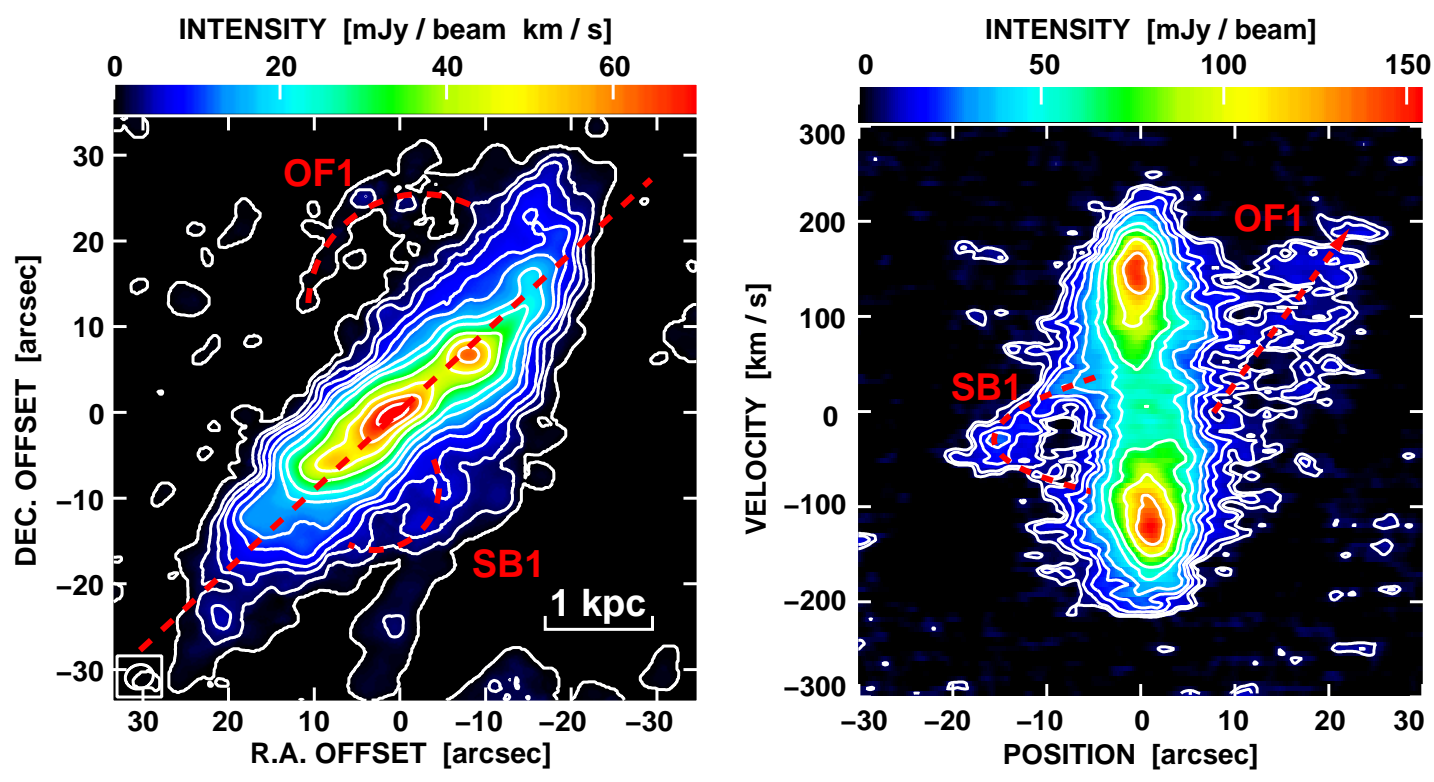

Figure 1: The NMA CO(1-0) data of NGC 2146 [एㄹ]. (Left) Integrated Intensity Map (moment 0). The central position corresponds to R.A. $=6^{\mathrm{h}} 18^{\mathrm{m}} 37.6^{\mathrm{s}}$, Dec. $=78^{\mathrm{d}} 21^{\mathrm{m}} 24.1^{\mathrm{s}}(\mathrm{J} 2000)$. The red-dotted line indicates the galactic disk. The red-dotted arcs indicate the superbubble (SB1) and outflow (OF1). (Right) The position-velocity ( $\mathrm{p}-\mathrm{v})$ diagram along minor axis. The red-dotted arc indicates the velocity of SB1. The red-dotted line indicates the velocity gradient of OF1.

molecular gas, while a similar structure appears in SB1 but the emission is weaker. In NGC 3628, ionized gas and molecular gas located at similar regions.

In order to realize the mechanism to push molecular outflows outward, we compared the ram pressure that the ionized outflow exerts on molecular outflow with the thermal pressure of ionized outflow. Table $\square$ lists the ram pressure and the thermal pressure in the two starburst galaxies. The result shows that the ram pressure is several orders higher than the thermal pressure. This indicates that the mechanism to push molecular outflows is mainly contributed by the shock of the supernova explosion rather than by the thermal expansion of hot gas.

Table 2: The pressure between molecular outflows and ionized outflows

\begin{tabular}{lccc}
\hline Galaxy & $\begin{array}{c}\log (\text { Ram pressure }) \\
{\left[\text { dyn cm } \mathrm{cm}^{-2}\right]}\end{array}$ & $\begin{array}{c}\log \text { (Thermal pressure) } \\
{\left[\mathrm{dyn} \mathrm{cm}^{-2}\right]}\end{array}$ & Reference \\
\hline NGC 2146 & $-(7-8)$ & $-(11-13)$ & [ए2] \\
NGC 3628 & $-(7-8)$ & $-(11-13)$ & [ए3] \\
\hline
\end{tabular}

\section{Summary}

We first provide the detail study of molecular superbubbles or outflows of two edge-on starburst galaxies, NGC 2146 and NGC 3628. We have used the NMA CO(1-0) observation data and the CXO soft X-ray archival data. From these data, we have two conclusions: (I) The sub- 

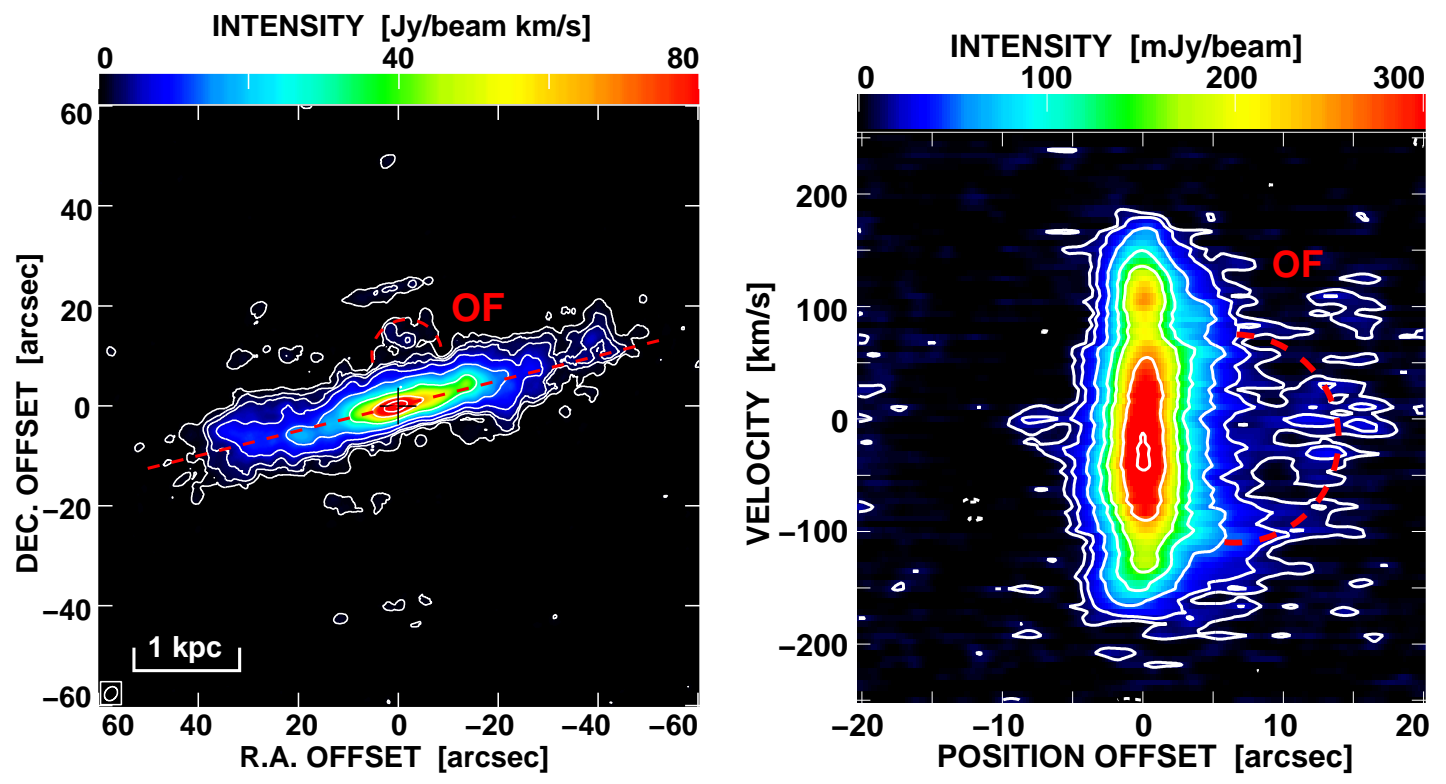

Figure 2: The NMA CO(1-0) data of NGC 3628 [[1]]]. (Left) Integrated Intensity Map (moment 0). The central position corresponds to R.A. $=11^{\mathrm{h}} 20^{\mathrm{m}} 17.0 \mathrm{~s}$, Dec. $=13^{\mathrm{d}} 35^{\mathrm{m}} 20.1^{\mathrm{s}}(\mathrm{J} 2000)$. The red-dotted arc indicates the outflow (OF). (Right) The $\mathrm{p}-\mathrm{v}$ diagram along minor axis of NGC 3628. The red-dotted arc indicate the velocity of outflow $(\mathrm{OF})$.

kpc to kpc scale molecular superbubbles and outflows have expanding velocity of 40-200 $\mathrm{km} \mathrm{s}^{-1}$ and energy of $\sim 10^{54-55} \mathrm{erg}$. (II) The mechanism to push molecular outflows outward is mainly contributed by the shock of supernova explosion.

\section{References}

[1] S. Matsushita, R. Kawabe, H. Matsumoto, T. G. Tsuru, K. Kohno, K.-I. Morita, S. K. Okumura and B. Vila-Vilaró, Formation of a Massive Black Hole at the Center of the Superbubble in M82, ApJ 545 (2) 107 [astro-ph/0011071]

[2] N. Nakai, M. Hayashi, T. Handa, Y. Sofue, T. Hasegawa and M. Sasaki, A nuclear molecular ring and gas outflow in the galaxy M82, PASJ 39 (5) 685

[3] R. B. Tully, Nearby Galaxeis Catalog, Cambridge: Cambridge University Press

[4] D.B. Sanders, J. M. Mazzarella, D.-C. Kim, J. A. Surace and B. T. Soifer, The IRAS Revised Bright Galaxy Sample, AJ 126 (4) 1607 [astro-ph/0306263]

[5] R. Della Ceca, R. E. Griffiths, T. M. Heckman, M. D. Lehnert and K. A. Weaver, ASCA Observations of the Starburst-driven Superwind Galaxy NGC 2146: Broadband (0.6-9 KEV) Spectral Properties, ApJ 514 (2) 772 [astro-ph/9810417]

[6] A. Taramopoulos, H. Payne and F. H. Briggs, HI observations of the starburst galaxy NGC 2146, $A \& A 365360$ [astro-ph/0005370]

[7] J. J. Condon, M. A. Condon, G. Gisler and J. J. Puschell, Strong radio sources in bright spiral galaxies. II - Rapid star formation and galaxy-galaxy interaction, ApJ 252 (1) 102 

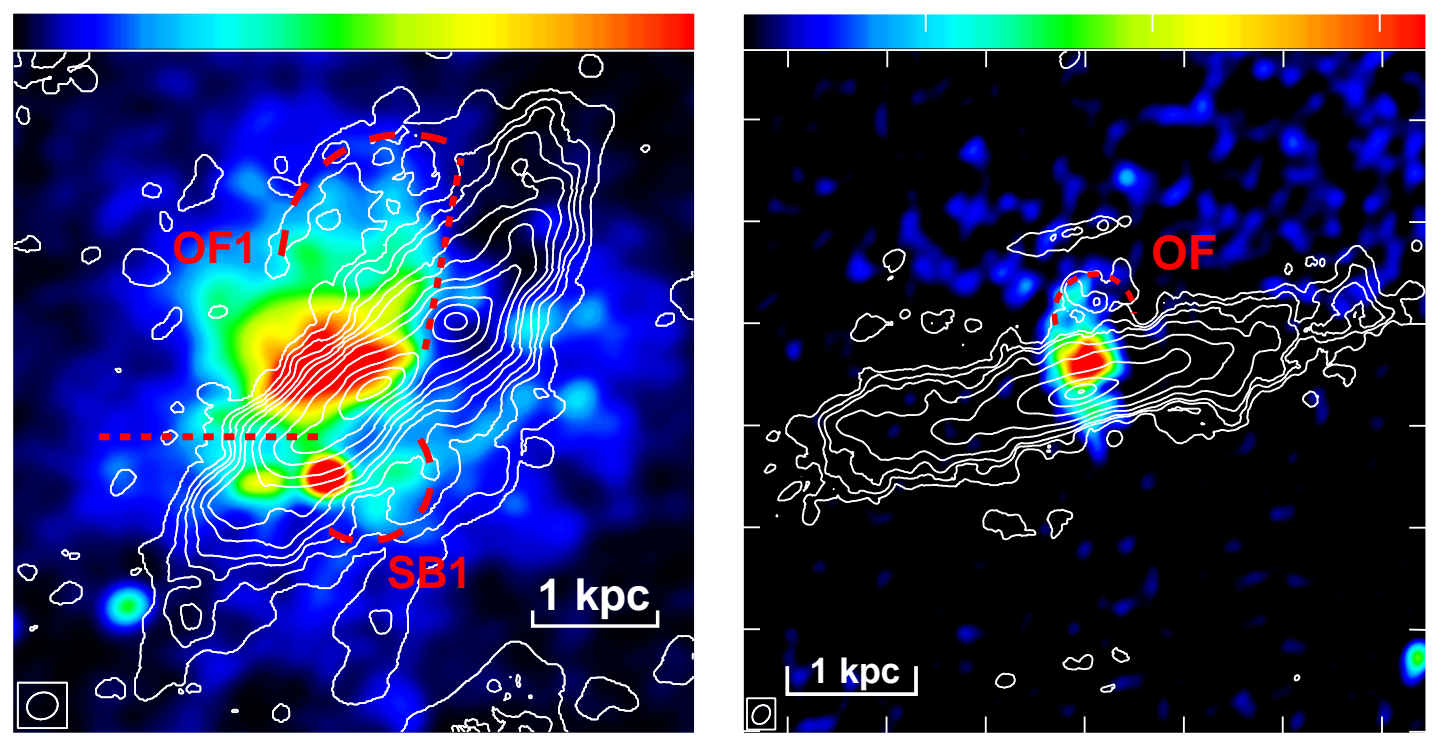

Figure 3: NMA CO(1-0) integrated intensity contour map overlaid on the Chandra soft X-ray image [U]] in color-scale. Left panel is NGC 2146 [ए2], and right panel is NGC 3628 [ए3]].

[8] D. K. Strickland, E. J. M. Colbert and T. M. Heckman, Another Intermediate-Mass Black Hole in a Starburst Galaxy? The Luminous X-Ray Source in NGC 3628 Reappears, ApJ 560 (2) 707 [astro-ph/0107115]

[9] D. K. Strickland, T. M. Heckman, E. J. M. Colbert, C. G. Hoopes and K. A. Weaver, A High Spatial Resolution X-Ray and Ho Study of Hot Gas in the Halos of Star-forming Disk Galaxies. II. Quantifying Supernova Feedback, ApJ 606 (2) 829 [astro-ph/0306598]

[10] J. A. Irwin and Y. Sofue, NGC 3628: The Nuclear Molecular Disk and Expanding Molecular Shells, ApJ 464738

[11] T. Inui, H. Matsumoto, T. G. Tsuru, K. Koyama, S. Matsushita, A. B. Peck and A. Tarchi, Chandra Observation of the Starburst Galaxy NGC 2146, PASJ 57 (1) 135 [astro-ph/0412249]

[12] A.-L. Tsai, S. Matsushita, K. Nakanishi, K. Kohno, R. Kawabe, T. Inui, H. Matsumoto, T. G. Tsuru, A. B. Peck and A. Tarchi, Molecular Superbubbles and Outflows from the Starburst Galaxy NGC 2146, PASJ 61 (2) 237 [astro-ph/0812.2828]

[13] A.-L. Tsai, S. Matsushita, A. K. H. Kong, H. Matsumoto and K. Kohno, First Detection of a Subkiloparsec Scale Molecular Outflow in the Starburst Galaxy NGC 3628, ApJ 752 (1) 38 [astro-ph/1204.3414] 